\title{
Achilles Tendon Rupture: Advances in Functional Rehabilitation and the Role of Non-operative Management
}

\author{
Forlizzi Julianne $\mathbf{M}^{*}$ and Swan Kenneth G \\ Department of Orthopedics, USA
}

*Corresponding author: Forlizzi Julianne M, Department of Orthopedics, Rutgers-Robert Wood Johnson Medical School, RWJ Place, MEB 422, New Brunswick, NJ 08901, USA

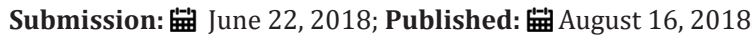

\begin{abstract}
Achilles tendon ruptures are a frequently seen orthopedic injury with an incidence of 18/100,000 individuals yearly. Achilles tendon ruptures (ATR) occur more frequently in males in the 4th-5th decade during athletic activities, but can also result from simple trauma. Previously, surgical management was recommended for ATR based on high rates of re-rupture with non-operative treatment. This paper explores recent level one and two randomized-control trials and systematic reviews, especially those including functional rehabilitation, to explore treatment options for acute Achilles tendon ruptures in the orthopedic setting. The data suggest a viable role for non-operative management with satisfactory functional results.
\end{abstract}

\section{Introduction}

Achilles tendon rupture is a potentially devastating event for a patient, especially a young, active one. In a clinical setting, the diagnosis is usually straightforward. Despite an uncomplicated diagnosis, treatment options can differ widely. In order to review differences between treatment options, an online literature review was conducted for the search terms "Achilles tendon rupture functional rehabilitation" "acute Achilles tendon rupture" by two authors independently. Randomized controlled trials, Cochrane reviews, and systematic reviews/meta-analyses published in the last twenty-five years (i.e. since 1992) were included. Twentyseven articles were identified that met inclusion criteria: studies published in the English language, those that treated acute Achilles tendon rupture, and those that employed a functional rehabilitation program (i.e. compared groups with accelerated range of motion and/or weight bearing). In studies that included an operative cohort, both minimally invasive and traditional open techniques were employed. In those that immobilized patients after injury, casts, splints, and other orthotics were included. Data on re-rupture was also included.

Exclusion criteria were duplicate studies, non-English articles, and studies treating patients with sub-acute or delayed presentations (greater than 3 weeks post-injury). Data was then extracted by the authors, together, and conclusions were drawn based on the following criteria:

1. Comparing operative to nonoperative treatment of acute Achilles tendon rupture,

2. Comparing outcomes of patients who were allowed early weight bearing with or without repair
3. Comparing outcomes of patients who were allowed early range-of-motion with or without repair

Combinations of cohorts who were allowed both early weightbearing and early range-of-motion were also analyzed.

The Achilles tendon rupture usually occurs during highenergy/jumping athletic activity [1]. Diagnosis is usually made clinically based on detailed history and physical exam with a positive Thompson test and/or palpable gap in the tendon. It is important to note that retraction occurs no further than the level of the gastrocnemius/soleus aponeurosis, from where the tendon arises [2]. Radiographs are performed to rule out other injury, including calcaneal avulsion fracture. Ultrasound or MRI may be used in the presence of a swollen lower extremity to confirm a complete rupture of the tendon if the diagnosis is in question $[2,3]$. The prevalence of Achilles tendon rupture (ATR) lies between 1020 per 100,000 adult individuals and is reported to be increasing [2]. Patients are typically male in the fourth decade, but women in that age group are susceptible to rupture as well. These injuries are most typical during racket sports or basketball, where the tendon is subject to tensile loads $10 \mathrm{x}$ a person's body weight [2]. Highlevel athletes are therefore susceptible to ATR, and this group often opts for operative management. Return to sport is more important in these cohorts, and operative repair is thought to aid complete recovery with suture fixation. It is also possible to rupture during everyday activity such as slipping with sudden, unexpected ankle dorsiflexion $[1,4]$. It is postulated that the mid-substance of the tendon is most prone to rupture because of its hypovascularity, an area that is defined as $2-6 \mathrm{~cm}$ proximal to its calcaneal insertion 
$[4,5]$. Some have hypothesized that tendon degeneration plays a major role in weakening, and eventual rupture of the Achilles, though one study found that only $5 \%(n=9)$ of their 176-person cohort had prior symptoms of pain [4]. Other risk factors for ATR include obesity, rheumatologic disease, neuropathy, recent steroid or fluoroquinolone use, and "weekend warrior" activity. Unfortunately, many published randomized controlled trials exempt patients with these risk factors (i.e. omit patients with diabetes) in order to demonstrate favorable outcomes of surgery and adequately compare patients undergoing both operative and non-operative interventions [6-8]. While standardizing the literature, the lack of data on this subset of patients ignores a significant portion of those suffering from an ATR and therefore leaves a gap in the clinical knowledge of how to treat these patients. Furthermore, studies comparing operative versus nonoperative intervention often have deviating rehabilitation protocols, making a direct comparison challenging. With more recent publications including quality levels of evidence, it is the authors' pursuit to investigate the recent successes of non-operative management of this injury especially in terms of allowing weight bearing and range of motion, and whether recent advances can serve to rehabilitate patients towards more successful outcomes (Figure 1).

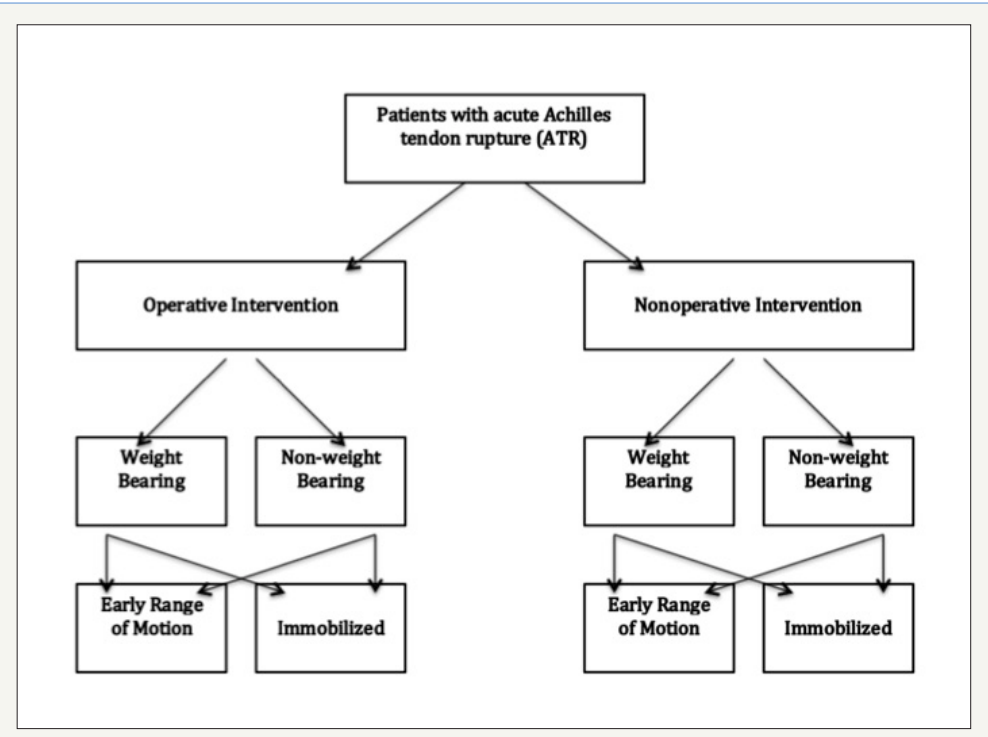

Figure 1: Scheme of possible rehabilitation strategies following diagnosis of acute achilles tendon rupture.

\section{Literature Review: Operative Intervention}

The most recent clinical practice guidelines from the AAOS were published in 2010 and include: both surgical and non-surgical management of acute Achilles tendon ruptures are graded with a "weak" recommendation [5]. That is, one cannot be superior to the other based on available evidence. Furthermore, a consensus guideline mentions that surgery should be cautionary in patients "with diabetes, neuropathy, an immune compromised state, age > 65 years, peripheral vascular disease, local/systemic dermatologic disorders, and/or who exhibits a sedentary lifestyle, uses tobacco, or is obese [5]." These warnings serve to remind orthopaedic surgeons of the nature of complications, especially those of skin compromise, infection, and rerupture.

When deciding on an operative treatment plan, discussion of risks and benefits of surgery are often based on literature published supporting a lower re-rupture rate in surgical cohorts as compared to non-operatively treated patients [9]. However, newer literature that combines early mobilization with either surgical or nonsurgical treatment does not distinguish clinically significant rerupture rates [10-12]. Functional outcomes, such as earlier return to work, must then be balanced with the risk of complications after surgery including sural nerve injury or local infection $[13,14]$.
Further, should medical risk stratification, pre-operative DVT, or other complication delay surgery, an equinus posterior splint may help in preventing retraction of the tendon $[13,14]$.

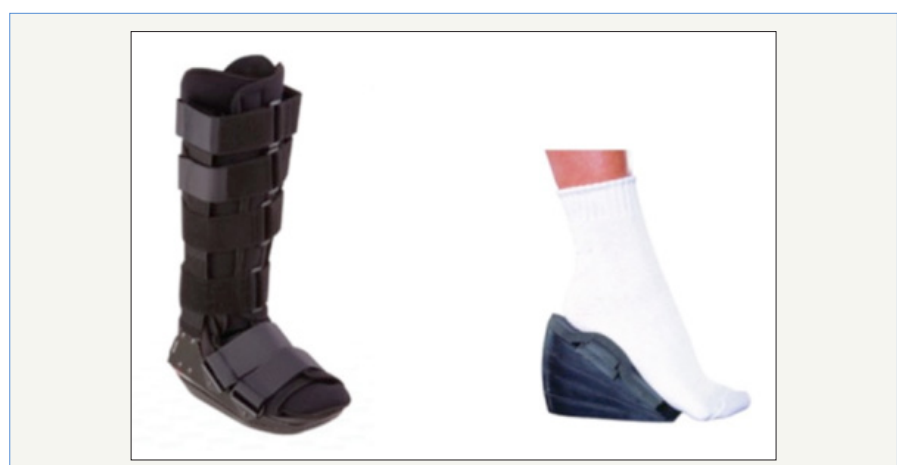

Figure 2

2a: An example of a walking boot for Achilles tendon rupture, in which progressive heel lifts can be placed to acquire the desired amount of equinus.

2b: Internal heel lift portion of the brace. Product made by Bledsoe, found on braceshop.com.

The most recent literature suggests that the most important determinate of post-rupture function lies in the rehabilitation 
protocol and functional bracing, which is much different than the traditional method of immobilizing following Achilles rupture (Figure 2). The AAOS recommendations, of moderate strength, include protected weight bearing as well as protected range-ofmotion despite their lack of recommendations for the operation itself $[13,14]$. These data point to the rehabilitation as the most vital part of recovery after ATR.

\section{Comparison of operative versus non-operative management}

The general belief is that operative repair is the preferred management option if complications can be avoided. In the literature, these "complications" range from temporary sural nerve paresthesias to reruptures to deep soft tissue infections: the definition has not been standardized. Most trials in the literature use re-rupture as a primary end point and therefore the most imperative complication to be avoided. A 2002 meta-analysis was first to quantify the relative risk of non-operative treatment as a tenfold rate of rerupture, from $1.3 \%$ to nearly $13 \%$. Bhandari [9] reviewed six previously published studies including over 400 patients and gleaned that "for every ten patients treated with surgery, one re-rupture is prevented. Conversely, for every 21 patients operated on one infection occurs. These data are often cited by orthopaedic surgeons when discussing management options with their patients. Further Cochrane Review by Khan [15] in 2010 found similar results with a 5\% re-rupture rate in surgical patients versus $12 \%$ in nonoperative patients with a $\mathrm{P}=0.00619$ Their conclusions echoed the 2002 study regarding re-rupture: it is much less likely in patients treated surgically, but produces significantly higher risk of complications such as wound infection.

\section{Remarkable flaws in these reviews include}

1. The variety of methods by which Achilles repairs occur and

2. Variability in the post-operative protocols, such as duration of immobilization and onset of weight bearing

Surgical options for ATR include percutaneous or open techniques [16]. With either approach a variety of options exist regarding placement of the incision and method of suture repair, thereby affecting patients differently. These variations are grouped together in outcome studies. Despite the conventional teaching that re-ruptures is a relatively common complication of conservative management even large, randomized studies failed to reach this conclusion to statistical significance [16]. Certain study such as Moller's demonstrated "equally good functional results if complications were avoided [17]. However, the rate of re-rupture after nonsurgical management is unacceptably high." This study, despite finding a ten-fold increase in reruptures with non-operative management, compared an operative group that employed functional rehabilitation to a conservative group who used a conventional plaster splint and was restricted from weightbearing. In the surgical group, both range of motion and weightbearing were allowed before the non-operative group was allowed to bear weight. Even with the bias inherent to the study design that favored early function in the operative cohort, both groups had more than half of participants return to athletics at the same level as before the injury.

A 1993 study in AJSM focused on operative versus nonoperative management of acute tears with one year of follow up and concluded that operative treatment was "preferable, but nonoperative management is an acceptable alternative [18]." Despite their findings that patients treated with surgery had a higher rate of return to sports, less calf atrophy, and fewer complaints at one year, the difference in major complication rate was not statistically significant. Minor complications were significantly lower in the non-operative group. Their study revealed seven reruptures out of the 55 tendons treated conservatively; however, there were three reruptures and two deep infections in the 56-person operative group.

Similarly, Metz [19] compared a total of 83 patients between nonoperative and minimally invasive surgery with one-year follow up and found that the differences in complications, namely re-ruptures, "appears to be clinically important...but not statistically significant [20]." They experienced 5 re-ruptures in the non-operative group and 3 in the operative. This level 2 study also employed the use of a sealed brace in the non-operative group only, which artificially elevated the complication rate in the non-operative group by inflating the number with "skin-related complications" including blisters from brace wear. No deep tissue infections were found in either group. More recently, a 2011 trial compared 80 patients including an operative group that was allowed weight-bearing at 6 weeks and a non-operative group allowed partial weight-bearing at 8 weeks and full weight-bearing at ten weeks [15]. The reruptures were not statistically significant with 2 in the operative group and 4 in the non-operative group. Three infections occurred in the operative group and none in the non-operative group. By one year, functional results were equivalent. They were "unable to show a convincing benefit for operation."

A transformation in published literature around the turn of the century demonstrated a shifting paradigm in management of Achilles tendon ruptures. Functional rehabilitation became more common and studies reflected this change. Altered protocol included such dynamic changes as immediate weight bearing in both operatively and non-operatively treated ruptures. Others have integrated early range of motion with or without weight-bearing. A standardized functional rehabilitation protocol, though, does not yet exist.

Two Level I studies published in the last decade found similar results when comparing operative and non-operative treatment using functional rehab. The first, published by Helander [21] found that at one year, both operative and non-operative groups had less function than their contralateral, uninjured side; however, early mobilization proved beneficial, and there were no statistically significant differences between groups regarding re-rupture rates [6]. The second, published by Olsson [6] in 2013 also found that 
their re-rupture rate in the non-operative group did not reach statistical significance [7]. Further, there were six complications in 49 operative patients that eventually resolved. They concluded that: "operation is successful, but not superior to non-operative management in quality of life, activity, or functional level." This finding may shift a practitioner's thinking when counseling patients in the office, who often expect surgery. A Level I study by Willits demonstrated 13 total complications in their operative group $(n=72)$ and only 6 in the non-operative group $(n=72)$ [12]. Their accelerated rehabilitation consisted of using a removable walking boot in approximately 20 degrees of plantarflexion during recovery. Re-ruptures ( 2 and 3, respectively) were not statistically significant. The results of this study echoed recent literature in that accelerated functional rehab proved beneficial. In their non-operative group "all measured outcomes... were acceptable and clinically similar to operative management." It was important to note that this study excluded insulin-dependent diabetics as well as patients with significant peripheral vascular disease. Similarly, Garrick [22] study found similar rates of re-rupture in operative and nonoperative patients over its two-year follow up while asserting that recovery was similar between the two groups as long as functional rehabilitation was applied [23].

A meta-analysis published in JBJS in 2012 evaluated 10 studies comparing operative and non-operative treatment of Achilles ruptures [24]. They found that if a patient underwent functional rehabilitation, there was no significant difference in re-ruptures. If a patient did not undergo functional rehabilitation, the absolute risk reduction of re-rupture treated by the operation was $8 \%$. The conclusion from this analysis is that: "conservative treatment should be considered at an institution with functional rehabilitation. It offers a similar rate of re-rupture with a decrease in complications." This assertion is contradictory to those from not ten years earlier which advocated for operative treatment. The differences stem from a courageous attempt at acclimatizing a patient while allowing integrated weight-bearing and range of motion exercises as early as in the immediate post-rupture period.

Re-rupture is a feared complication of Achilles repair. Studies such as Cetti [18] suggest that ultrasound could be a useful technique in evaluating repair during the follow-up phases of nonop treatment to observe the secondary integrity of the tendon6. If the ends do not oppose, repeat operation may be considered. Unfortunately, this is not yet routine practice and the timing of the decision to pursue an operation has not been defined. To the authors' knowledge no current data suggests a distance between free tendon ends at which operation is mandatory.

Infection is a second complication that cannot be understated. In an aging and enlarging population, an infection in an area not immediately visible to a patient can be devastating. Skin coverage in this area is also not ideal, especially incorporating the degree of swelling that can be present after such an injury; coverage following formal debridement(s) may require flaps or skin grafting. Vander Eng [25] in 2013 reviewed 7 level one studies comparing operative and nonoperative treatment. They found two times the amount of complications in the surgical group. Combining this data from the earlier published data of nearly 1 in 20 patients acquiring a complication (usually infection), physicians should be wary to offer an operation to a patient, especially given comparable results with nonoperative management.

Bruggeman and Turner went so far as to classify the risk factors for wound complications [26]. In their study, they identified tobacco use as the single most important factor for development of wound infection. Steroid use and female gender were also significantly associated with this complication. Though age $>50$, delayed repair $>28$ days post-injury, and obesity (defined as BMI $>30$ ) were correlated with increased rate of infection, the association was not statistically significant. Overall the authors derived that over $40 \%$ of patients with at least one of the following: diabetes, tobacco use, and/or steroid use, suffer from a post-operative infection and caution physicians to consider this data when deciding on treatment for a patient with ATR.

\section{Regarding literature focused on weight-bearing versus non-weight bearing}

Certain published studies focus on a smaller subset of patients, i.e. whether weight bearing or not affects outcomes after Achilles tendon rupture (Figure 1). While some of these trials employ different experimental groups, direct comparisons cannot always be drawn from their conclusions because of the different trial protocols.

Following an operation, Costa and colleagues placed a carbon fiber orthosis with heel-lifts on patients immediately postoperatively and compared these patients to patients casted in a formal plaster cast for 8 weeks $[27,28]$. The orthosis group was allowed to weight bear and range of motion was encouraged. The casted group experienced serial cast changes at two-week intervals but was not allowed weight bearing nor were they allowed range of motion. Their patients who bore weight experienced much better outcomes with 6 fewer weeks to return to normal walking and 9 fewer weeks for stair climbing. There were no differences between groups regarding return to work or to sports. The two reruptures in the weight-bearing group were attributed to lack of adherence to protocol, with at least one patient attempting to train on a treadmill. The authors of this study advocate immediate weight bearing post-operatively (as well as appropriate patient selection and compliance with post-op protocol).

Suchak [24] performed a Level I trial in 110 patients after surgical repair. The primary end point was quality-of-life. Secondary end points included strength, activity, return to work or sport, and complications. Two weeks following the surgery, half of the patients were randomized to a weight-bearing group in a $20^{\circ}$ plantarflexed boot (Figure 2) or non-weight bearing group for four more weeks to total 6 weeks of non-weight bearing. Neither group experienced any reruptures nor did the authors find that weight bearing had any detrimental effect on recovery. This study was paramount in demonstrating that weight bearing post-operatively can be a safe means of rehabilitation. 
Similar weight-bearing versus non-weight bearing studies were performed in patients treated non-operatively. Costa's abovementioned study was divided into two arms: the second, nonoperative arm had a slightly different design than the operative arm. This group used carbon fiber orthosis with heel-rasies for 6 weeks and then reduced the plantarflexion gradually over the next twelve weeks $[27,28]$. Again, this group was encouraged to range the ankle. The non-weight bearing group endured a short leg cast in plantarflexion for 6 weeks then had serial casts with reduced plantarflexion for a total of 12 weeks of study time. This study revealed no difference in time to normal walking or stair climbing, nor was there a difference in return to work or sports. There was "no evidence of functional benefit from weight-bearing, but practical advantages did not predispose patients to a higher complication rate." Therefore, they advocated for immediate weight bearing in non-operative patients as well.

Young [12] published a Level I study in 2014 comparing nonoperative Achilles ruptures that were casted for 8 weeks. Though there was less subjective stiffness in the weight-bearing group, there were no other statistically significant differences, including in the rate of re-rupture. The authors concluded that weight bearing outcomes "are at least equivalent." Because the overall re-rupture rate was low (3 out of 84 patients) they continue to support non-op management of Achilles tendon ruptures.

A comprehensive summary of weight-bearing versus nonweight bearing post-op protocols is found in Brumann's [29] 2014 meta-analysis that focused on five level one studies. They found that weight-bearing protocols were superior in satisfaction, in earlier ambulation, and in return to previous activity (there was no significant difference in re-rupture rates). Their conclusions were to allow full weight-bearing post-operatively for these reasons.

Table 1: Example of rehabilitation protocol, adapted from Twaddle \& Poon [8].

\begin{tabular}{|c|c|}
\hline Time Post-op & Activity Allowed \\
\hline Days $0-14$ & Equinus cast, no weight-bearing or range-of-motion \\
\hline Week 2-3 & Non-weight bearing $30^{\circ}$ equinus in AAFO boot; may remove $5 \mathrm{~min} / \mathrm{hr}$ for ROM exercises \\
\hline Week 3-4 & Non-weight bearing $20^{\circ}$ equinus \\
\hline Week 4-5 & Non-weight bearing $10^{\circ}$ equinus \\
\hline Week 5-6 & Non-weight bearing $0^{\circ}$ equinus \\
\hline Week 6+ & Weight-bearing as tolerated; remove boot for sleep \\
\hline Week 8+ & Remove boot for strengthening exercises \\
\hline
\end{tabular}

Protocol available at: https://www.sportsmed.net.nz/foot_and_ankle

Long-term follow up was published in 2015 regarding comparing ROM and bracing. Specifically, a study evaluated 50 patients eleven years post-operatively. Initial treatment included either a brace for 6 weeks with the ankle allowed to range between neutral or plantar-flexion compared with a group that was immobilized in a traditional cast for the same amount of time. No significant differences were found in functional scores, and both

\section{Regarding literature focused on range of motion versus no range of motion}

A smaller subset of literature exists comparing effects of early range of motion (ROM) following an Achilles tendon rupture. Few studies address this issue following operative repair of the tendon. One study compared 71 patients with immediate range of motion in a walking brace to casted patients who were not allowed any ROM for 8 weeks [20]. The brace included an elastic pulley to allow for active dorsiflexion, but at rest had the ankle in 30 degrees of equinus. The authors concluded that early, restricted motion appears to shorten the time to rehabilitate the injury [17]. There were no complications found related to early range of motion as there was one re-rupture (failed repair) in the ROM group and two in the immobilized group. Notable conclusions from this study include that the early motion group had a significantly earlier return to work by approximately 25 days ( 43 vs. 68 with $\mathrm{p}<0.05$ ).

In New Zealand, Twaddle \& Poon [8] engineered a Level I study comparing ROM versus no range of motion in both an operative group of 20 patients and non-operative group of 22 patients, both with one-year follow up. This randomized study found similar results to Mortenson [17] earlier work. There were two reruptures in the operative group (patients from which were immobilized until post-operative day 10; weight-bearing was allowed at 6 weeks post-op) and one in the non-operative group (which had the same protocol-immobilization until day 10). There were neither differences in plantar or dorsiflexion nor any differences in calf circumference after one year. Based on this evidence, the authors concluded that early range of motion is a safe and acceptable form of rehabilitation (Table 1). There were comparable functional results and low re-rupture rates. They stated that controlled, early range of motion was the most important part of treatment rather than operation. 
should not come as a surprise given the previously published data on flexor tendon healing in the hand. As early as the 1980's, Gelberman et al. [23] performed studies on early passive mobilization on repaired flexor tendons in canine models. Those tendons allowed motion healed with less adherence, less scar tissue, and increased strength over all time points studied [7,23]. These results are likely based on the histobiology of the surrounding paratenon and the ability of the incorporated tendon to glide through it. Their conclusions were based on "flexor sheath continuity", analogous to the Achilles tendon and its function as a flexor of the lower extremity. Early ROM may allow for proper reorientation of tendon fibrils that would otherwise remain disorganized and scar in an immobilized ankle.

In summary, the following data have been published with a grade "A" recommendation:

1. Diagnosis of ATR is based on patient history and physical exam

2. Operative repair of ATR may provide functional benefits, but these may be transient

3. Higher re-rupture rates previously associated with nonoperative management of ATR have been lowered with functional rehabilitation protocols [31-38]. It is the authors' practice to offer nonoperative management of ATR to our own patients.

The most recent prospective, randomized controlled trials are employing functional rehabilitation guidelines in both postoperative patients and non-operative patients. This strategy finally enables readers to glean a true comparison between groups, rather than to separate out comparable data between groups. Studies published as recently as 2016 indicate that functional rehabilitation is viable for nonsurgical patients, but a significant difference in calf muscle strength was found in the operative treatment group [13]. These data may be extrapolated in the future for amateur, and possibly professional, athletes looking to return to a competitive level of sport. Their focus was on the first year following injury, when most of the muscle regeneration occurs, relying on better strength in the short term to predict better endurance in the long term.

\section{Conclusion}

When treating a patient with an acute ATR, overall considerations must take the patient's lifestyle into account. Their functional status, wound healing potential, and desire to cooperate in a treatment plan are all paramount to successful outcomes. The studies reviewed often omitted diabetic, neuropathic, or obese patients entirely. These patients constitute a large part of the general practice and cannot be ignored; however, there is insufficient data to refer to when attempting to counsel them. At this point, we can only present a synthesis of published data and decide together on the best course of action to regain function.

\section{References}

1. Anagnostopoulou S, Mavridis I (2013) Achilles' death: anatomical considerations regarding the most famous trauma of the trojan War. J Trauma Acute Care Surg 74(3): 946-947.

2. Longo U, Ronga M, Maffuli N (2009) Acute ruptures of the achilles tendon. Sports Med Arthrosc Rev 17(2): 127-138.

3. Chiodo CP, Guss DG, Smith JT (2015) Acute achilles tendon rupture: a critical analysis review. JBJS Reviews 3(4).

4. Maffulli N, Tallon C, Wong J, Lim K, Bleakney R (2003) Early weight bearing and ankle mobilization after open repair of acute midstubstance tears of the achilles tendon. Am J Sports Med 31(5): 692-700.

5. Chen TM, Rozen WM, Pan WR, Ashton MW, Richardson MD, et al. (2009) The arterial anatomy of the achilles tendon: anatomical study and clinical implications. Clin Anat 22(3): 377-385.

6. Olsson N, Silbernagel KG, Eriksson BI, Sansone M, Brorsson A, et al. (2013) Stable surgical repair with accelerated rehabilitation versus nonsurgical treatment for acute Achilles tendon ruptures: a randomized controlled study. Am J Sports Med 41(12): 2867-2876.

7. Savio LY, Woo RH, Gelberman NG, Cobb D, Amiel KL, et al. (1981) The importance of controlled passive mobilization on flexor tendon healing: a biomechanical study. Acta Orthopaedica Scandinavica 52(6): 615-622.

8. Twaddle BC, Poon P (2007) Early motion for Achilles tendon ruptures: is surgery important? A randomized, prospective study. Am J Sports Med 35(12): 2033-2038.

9. Bhandari M, Guyatt GH, Siddiqui F, Morrow F, Busse J, et al. (2002) Treatment of acute achilles tendon ruptures: a systematic overview and metaanalysis. Clin Orthop Relat Res (400): 190-200.

10. Jones MP, Khan R, Smith CR (2012) Surgical interventions for treating acute achilles. J Bone Joint Surg Am 94(12): e88.

11. Wallace RG, Heyes GJ, Michael AL (2011) The non-operative functional management of patients with a rupture of the tendo Achillis leads to low rates of re-rupture. J Bone Joint Surg Br 93(10): 1362-1366.

12. Young SW, Patel A, Zhu M, van Dijck S, McNair P, et al. (2014) Weightbearing in the nonoperative treatment of acute achilles tendon ruptures: a randomized controlled trial. J Bone Joint Surg Am 96(13): 1073-1079.

13. Lantto I, Heikkinen J, Flinkkila T, Ohtonen P, Siira P, et al. (2016) A Prospective randomized trial comparing surgical and nonsurgical treatments of acute achilles tendon ruptures. Am J Sports Med 44(9): 2406-2414.

14. Chiodo CP, Glazebrook M, Bluman EM, Cohen BE, Femino JE, et al (2010) American academy of orthopaedic surgeons. american academy of orthopaedic surgeons clinical practice guideline on treatment of achilles tendon rupture. J Bone Joint Surg Am 92(14): 2466-2268.

15. Khan RJ, Smith CRL (2010) Surgical interventions for treating acute achilles tendon ruptures. Cochrane Database Syst Rev (9): CD003674.

16. Willits K, Amendola A, Bryant D, Mohtadi NG, Giffin JR, et al. (2010) Operative versus nonoperative treatment of acute Achilles tendon ruptures: a multicenter randomized trial using accelerated functional rehabilitation. J Bone Joint Surg Am 92(17): 2767-2775.

17. Mortensen N, Skov O, Jensen PE (1999) Early motion of the ankle after operative treatment of a rupture of the Achilles tendon. A prospective, randomized clinical and radiographic study. J Bone Joint Surg Am 81(7): 983-990. 
18. Cetti R, Christensen SE, Ejsted R, Jensen NM, Jorgensen U (1993) Operative versus non-operative treatment of achilles tendon rupture. A prospective randomized study and review of the literature. Am J Sports Med 21(6): 791-799.

19. Metz R, Verleisdonk EJ, Heijden VGJ, Clevers GJ, Hammacher ER, et al. (2008) Acute achilles tendon rupture: minimally invasive surgery versus nonoperative treatment with immediate full weightbearing-a randomized controlled trial. Am J Sports Med 36(9): 1688-1694.

20. Möller M, Movin T, Granhed H, Lind K, Faxén E, et al. (2001) Acute rupture of tendon achillis. A prospective randomised study of comparison between surgical and non-surgical treatment. J Bone Joint Surg 83(6): 843-848.

21. Helander NK, Silbernagel KG, Thomeé R, Faxén E, Olsson N, et al (2010) Acute achilles tendon rupture: a randomized, controlled study comparing surgical and nonsurgical treatments using validated outcome measures. Am J Sports Med 38(11): 2186-2193.

22. Garrick JG (2012) Does accelerated functional rehabilitation after surgery improve outcomes in patients with acute Achilles tendon ruptures? Clin J Sport Med 22(4): 379-380.

23. Gelberman RH, Woo SL, Amiel D (1990) Influences of flexor sheath continuity and early motion on tendon healing in dogs. J Hand Surg Am 15(1): 69-77

24. Suchak A, Bostick G, Beaupre L, Durand D, Jomha N (2008) The influence of early weight bearing compared with non-weight-bearing after surgical repair of achilles tendon. J Bone Joint Surg Am 90(9): 1876-1883.

25. Eng DMV, Schepers T, Goslings JC, Schep NW (2013) Rerupture rate after early weight bearing in operative versus conservative treatment of achilles tendon ruptures: a meta-analysis. J Foot Ankle Surg 52(5): 622-628.

26. Bruggeman NB, Turner NS, Dahm DL, Voll AE, Hoskin TL, et al. (2004) Wound complications after open achilles tendon repair: an analysis of risk factors. Clin Orthop Relat Res (427): 63-66.

27. Costa M, Shepstone L, Darrah C, Marshall T, Donnell S (2003) Immediate full-weight-bearing mobilisation for repaired Achilles tendon ruptures: a pilot study. Injury 34(11): 874-876.
28. Costa ML, MacMillan K, Halliday D, Chester R, Shepstone L, et al. (2006) Randomised controlled trials of immediate weight-bearing mobilisation for rupture of the tendo Achillis. J Bone Joint Surg Br 88(1): 69-77.

29. Brumann M, Baumbach SF, Mutschler W, Polzer H (2014) Accelerated rehabilitation following Achilles tendon repair after acute rupture Development of an evidence-based treatment protocol. Injury 45(11): 1782-1790.

30. Lantto I, Heikkinen J, Flinkkila T, Ohtonen P, Kangas J, et al. (2015) Early functional treatment versus cast immobilization in tension after achilles rupture repair: results of a prospective randomized trial with 10 or more years of follow-up. Am J Sports Med 43(9): 2302-2309.

31. Cooper MT (2015) Acute achilles tendon ruptures: does surgery offer superior results (and other confusing issues)? Clin Sports Med 34(4): 595-606.

32. Barfod KW, Bencke J, Bauridsen HB, Ban I, Ebscov L, et al. (2014) Nonoperative dynamic treatment of acute achilles tendon rupture: the influence of early weight-bearing on clinical outcome: a blinded, randomized controlled trial. J Bone Joint Surg Am. 96(18): 1497-1503.

33. Holm C, Kjaer M, Eliasson P (2015) Achilles tendon rupture-treatment and complications: a systematic review. Scand J Med Sci Sports 25(1): e1-e10.

34. Kearney R, Costa M (2012) Current concepts in the rehabilitation of an acute rupture of the tendo Achillis. J Bone Joint Surg Br 94(1): 28-31.

35. Keating J, Will E (2011) Operative versus non-operative treatment of acute rupture of tendo Achilles. J Bone Joint Surg Br 93: 1071.

36. Kou J (2010) AAOS clinical practice guideline: acute achilles tendon rupture. J Am Acad Orthop Surg 18(8): 511-513.

37. Leppilahti J, Puranen J, Orava S (1996) Incidence of achilles tendon rupture. Acta Orthop Scand 67(3): 277-279.

38. Soroceanu A, Sidhwa F, Aarabi S, Kaufman A, Glazebrook M (2012) Surgical versus nonsurgical treatment of acute achilles tendon rupture: a meta-analysis of randomized trials. J Bone Joint Surg Am 94(23): 2136-2143.
Creative Commons Attribution 4.0 International License

For possible submissions Click Here

\section{Submit Article}

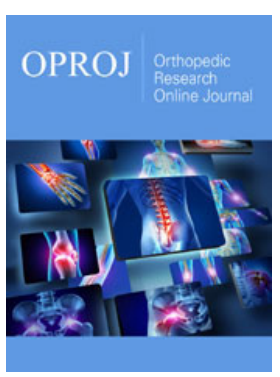

\section{Orthopedic Research Online Journal}

\section{Benefits of Publishing with us}

- High-level peer review and editorial services

- Freely accessible online immediately upon publication

- Authors retain the copyright to their work

- Licensing it under a Creative Commons license

- Visibility through different online platforms 